\title{
"Double Hit" Homozygous Mutations for Two Different Rare Inborn Errors of Metabolism: A Burden for Countries with High Prevalences of Consangineous Marriages
}

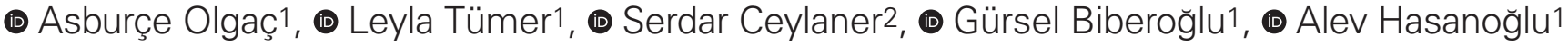 \\ ${ }^{1}$ Gazi University Faculty of Medicine, Department of Pediatrics, Division of Inborn Errors of Metabolism, Ankara, Turkey \\ 2Intergen Genetics Center, Clinic of Medical Geneticist, Ankara, Turkey
}

\begin{abstract}
Inborn errors of metabolism comprise a broad range of genetic diseases of which most are inherited in an autosomal recessive manner. Although being rare, there is a significant increase in their rate especially in countries where consanguineous marriages are performed. Isovaleric aciduria is an organic aciduria characterized by abnormal leucine metabolism resulting from a deficiency in the enzyme isovaleryl-CoA dehydrogenase. Niemann-Pick disease Type $\mathrm{C}$ is a rare autosomal recessive inherited disorder involving the intracellular transport of endocytosed cholesterol with sequestration of unesterified cholesterol in lysosomes and late endosomes. Both of these disorders are rarely encountered inborn errors of metabolism. We report a case of a boy with marked jaundice and hepatosplenomegaly, who was later diagnosed as isovaleric aciduria and Niemann-Pick disease Type C concomitantly. The diagnoses were proven by genetic analyses. A novel mutation for Niemann-Pick Type $\mathrm{C}$ has also been defined in this case report.

Keywords: Inborn erors of metabolism, Niemann-Pick Type C, isovaleric aciduria, consanguinity
\end{abstract}

\section{Introduction}

Inborn errors of metabolism (IEM) are a group of genetically inherited disorders involving the effects of enzymes of the body on metabolism. Although being rare, there is a significant increase in their rate, especially in countries where consanguineous marriages are performed (1). We report a case of a 2-month old boy who was diagnosed as Isovaleric aciduria and Niemann-Pick disease Type C (NP-C) concomitantly.

\section{Case report}

Two-month old boy was admitted due to jaundice and marked hepatosplenomegaly. He was the $3^{\text {rd }}$ child of consanguineous parents (first degree cousins). His prenatal, natal and family histories were uncomplicated. Jaundice had started when he was 5 days old and was still significant at the time of admission. Hepatosplenomegaly was detected in the outpatient clinic $8^{\text {th }}$ during the routine second month examination and he was referred to our center for further evaluation. His body weight was $5.2 \mathrm{~kg}$ (25 $5^{\text {th }}$ percentile) and height was $60 \mathrm{~cm}\left(50^{\text {th }}\right.$ percentile). Physical examination revealed yellowing of the skin and eyes, hepatomegaly [4 cm palpable below the lower costal margin ( $\mathrm{LCM})$ ] and splenomegaly (8 $\mathrm{cm}$ palpable below the LCM). Neurological examination showed normal findings. Complete blood count and peripheral blood smear showed normal results. Liver function tests revealed increased $\gamma$-glutamyl transferase (277 
$\mathrm{U} / \mathrm{L})$, alkaline phosphatase (473 U/L), conjugated bilirubine (5.25 mg/dL), serum glutamate pyruvate transaminase (SGPT) $64 \mathrm{U} / \mathrm{L}$, serum glutamate oxaloacetate transaminase (SGOT) $264 \mathrm{U} / \mathrm{L}$, with normal albumin and International Normalized Ratio (INR). Arterial blood gas analysis, serum lactate and ammonia were within normal limits. Abdominal ultrasonography showed hepatosplenomegaly. $\mathrm{He}$ was further evaluated by immunoglobulins, creatine kinase, amylase, thyroid function tests, alfa 1-antitripsine, ceruloplasmine, urine analysis and Benedict's test of urine which showed normal results. TORCH and hepatitis serology were negative. Chitotriosidase (165, normal range 0-120) and alpha feto protein (191.029 $\mathrm{ng} / \mathrm{mL}$, normal range $0-7)$ were elevated. Tandem mass spectrometric analysis using dried blood spots indicated an elevated isovaleryl carnitine level $(4 \mu \mathrm{mol} / \mathrm{L}$ [not reported (NR) $<0.6 \mu \mathrm{mol} / \mathrm{L}$ ]. Free carnitine level was within normal limits. His urine organic acid profile showed a markedly increased concentration of isovalerylglycine $[80 \mathrm{mmol} / \mathrm{mol}$ creatinine $(\mathrm{Cr})$, normal value: $0]$, and a normal concentration of 3-hydroxyisovaleric acid (6.5 mmol/mol Cr; NR: 0.7-14.4 mmol/mol Cr). In respect to the results of the metabolic tests, genetic testing with Next Generation Sequencing (Miseq-Illumina) for the IVD gene was performed using genomic DNA which was isolated from his peripheral leukocytes due to a suspicion of isovaleric acidemia (IVA). A previously reported p.A314V (c.941C>T) homozygous mutation was detected (Figure 1). Although a diagnosis was reached, the patient's splenomegaly still could not be explained and further investigation was essential. With this aim, bone marrow biopsy was performed which revealed macrophages with abnormal cholesterol storage (Figure 2). Lysosomal enzyme studies from leukocytes were carried out for glucocerebrosidase, sphingomyelinase and acid lipase enzymes which were $5.93 \mathrm{nmol} / \mathrm{h} / \mathrm{mg}$ protein (normal range $9.4 \pm 3.2$ ), $5.13 \mathrm{nmol} / \mathrm{h} / \mathrm{mg}$ protein (normal $7.73 \pm 3.08$ ) and $150 \mathrm{nmol} / \mathrm{h} / \mathrm{mg}$ protein (normal range $62.47 \pm 35.9$ ) respectively. Since these results made the condition unlikely to be as a result of Gaucher, NiemannPick disease Type A-B and Wolman diseases, further studies were performed including Next Generation Sequencing

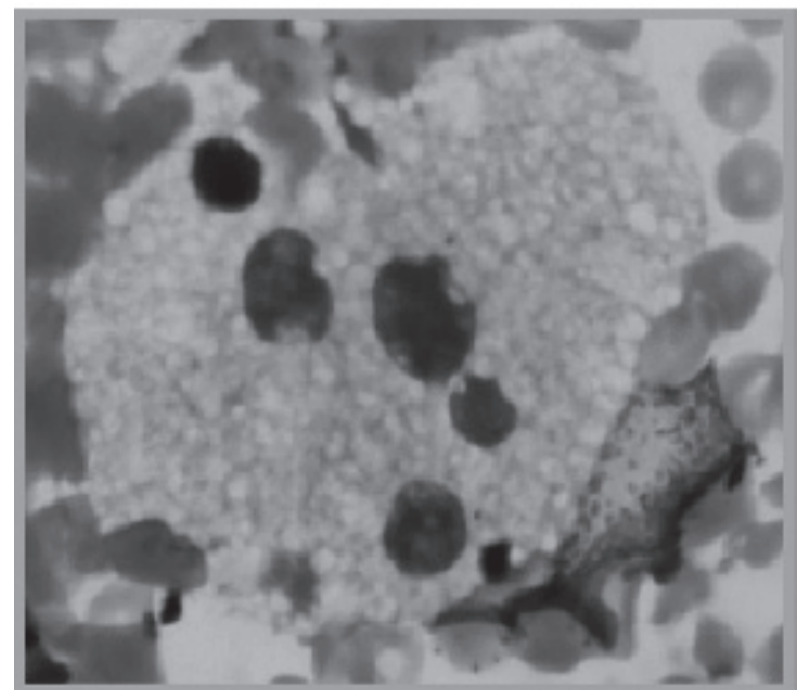

Figure 2. Bone marrow biopsy showing macrophages with abnormal cholesterol storage

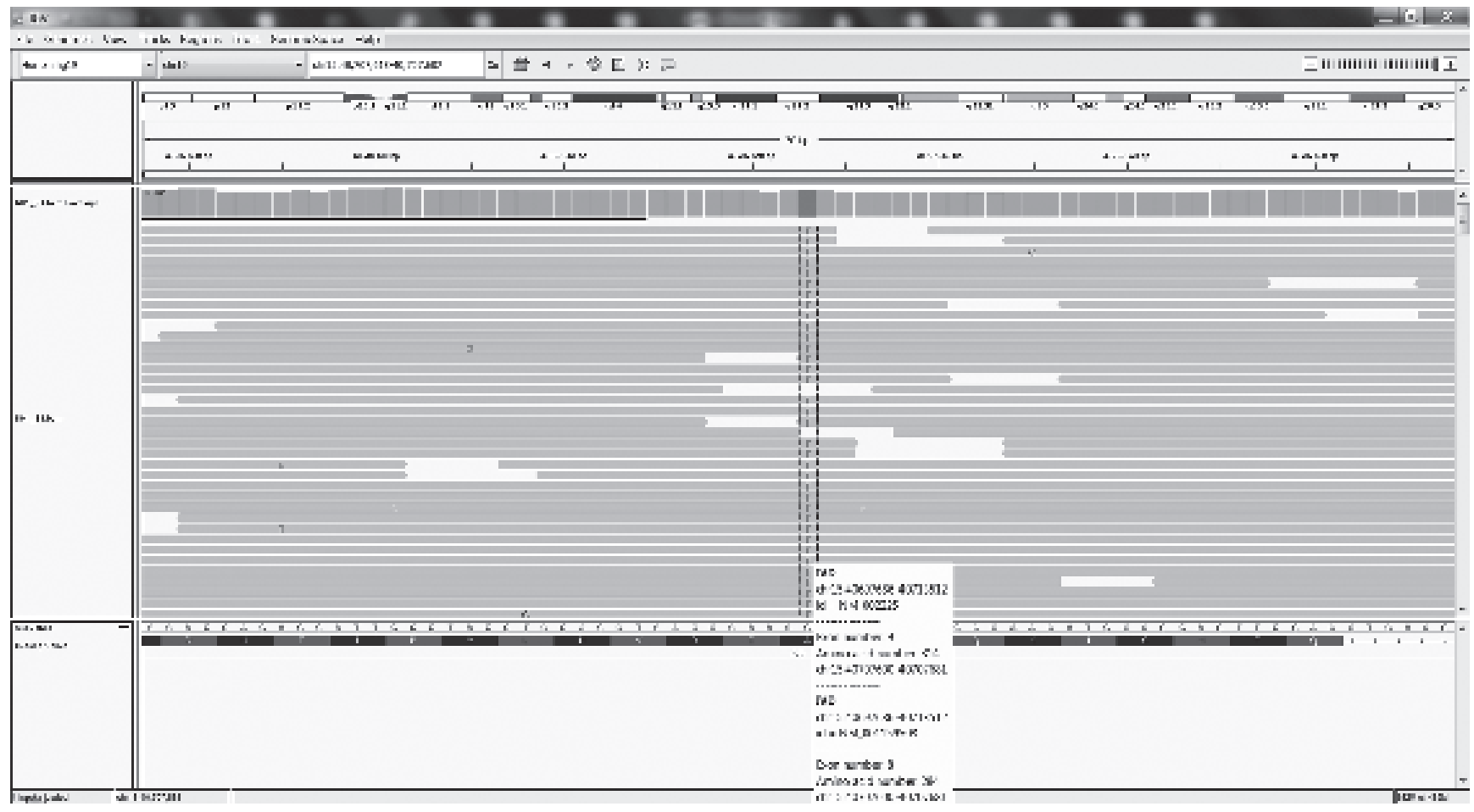

Figure 1. Sequence analysis for IVD gene 
of NPC1 gene, which was carried out on DNA isolated from leukocytes, for NP-C. A novel homozygous frameshift mutation in NPC1 gene: p.P733Sfs*10 (c.2196_2197insT) was detected (Figure 3 ). Both of the boy's parents were shown to carry both mutations. The patient had remained in a stable condition on glycine supplementation and low-leucine diet. After the age of 1 , mild psychomotor retardation, vertical supranuclear gaze palsy and mild spasticity were detected upon physical examination. Electroencephalogram showed irregular background activity with focal slowing. Miglustat therapy was initiated. His neurological condition remained stable after miglustat therapy. He died suddenly at home during an episode of aspiration pneumonia at the age of 2 .

\section{Discussion}

IEM comprise a broad range of genetic diseases of which most are inherited in an autosomal recessive manner. Individual IEM are rare, most having an incidence of less than 1 per 100.000 births, increasing with the prevalence of consanguineous marriages (2). The incidence of two different IEM occurring concomitantly in a patient has been rarely reported in literature. Guy et al. (3) reported Fabry disease and aspartylglucosaminuria in a patient with consangineous parents. The authors emphasized consanguinity as a factor in increasing the risk of autosomal recessive disorders occurring concomitantly (3). Concolino et al. (4) reported phenylketonuria and Fabry disease to co-exist in a 3-year old boy with consaguineous parents. IVA is a type of organic aciduria characterized by an abnormal leucine metabolism resulting from the deficiency in the enzyme isovaleryl-CoA dehydrogenase. The incidence is estimated to be 1 in $62.500-250.000$ (5). Currently, there are no published data about the incidence of IVA for Turkey. To date, more than 40 heterogeneous mutations in the IVD gene (Online Mendelian Inheritance in Man: 607036), located on chromosome 15q1415 (http://www.hgmd.org/) have been reported. Missense and splicing mutations are the most common. The p.A314V (c.941C>T) homozygous mutation in the IVD gene of our patient is a common frameshift mutation and has been previously reported with $20 \%$ residual enzyme activity (6). Although it is mostly accepted to cause a mild, asymptomatic clinic with normal development, it is still uncertain whether individuals have a risk of clinical manifestation. While these individuals may have normal leucine homeostasis under physiological conditions, their risk of metabolic decompensation under stress conditions remains to be determined (5). Nasser et al. (7) showed that the A314V (Also reported as A282V) mutant enzyme had thermal instability, a finding that might play a role in the development of symptoms during times of illnes. In our patient, initially, a low leucine diet was introduced with a further plan of transforming into a normal protein diet in further follow-up, since the risk of acute decompensation of IVA could not be ruled out completely, as it has been reported in the literature. The low leucine was continued until he was one year old. None of his febrile attacks had been accompanied by metabolic decompensation during this period. NP-C is a rare disorder of intracellular transport of endocytosed cholesterol with sequestration of unesterified cholesterol in lysosomes

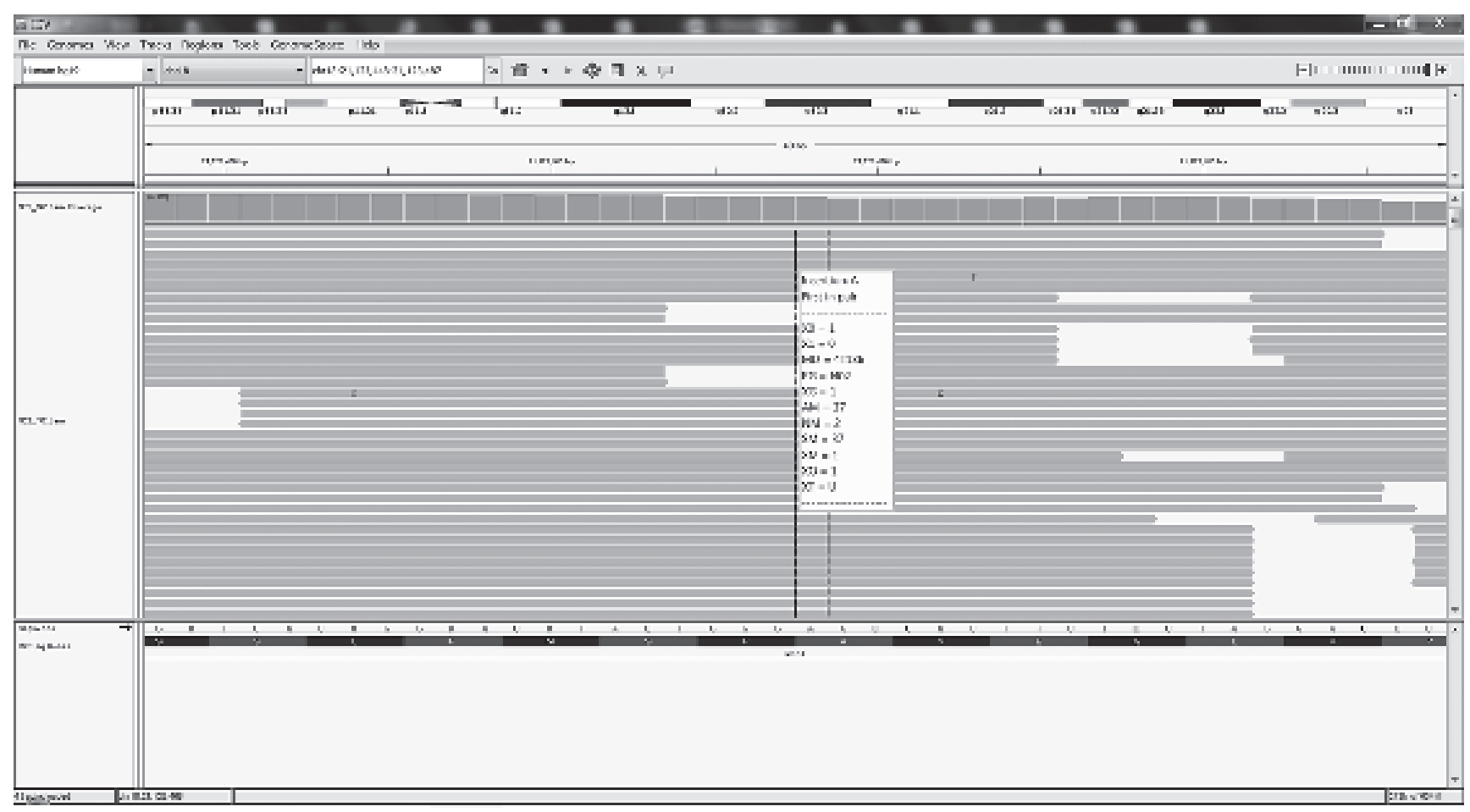

Figure 3. Sequence analysis for NPC gene 
and late endosomes. Most of its patients (95\%) have mutations in the NPC1 gene (8). The prevalence in Western Europe has been estimated to be approximately 0.0008 $0.0007 \%$ of live births. Again, there are no specific data for Turkey. To date, over 300 disease-causing mutations have been reported, most being missense mutations (9). Different clinical phenotypes of the disease can be categorized according to the patient's age at onset of signs. Perinatal presentation is characterized by a prolonged neonatal cholestatic icterus and progressive hepatosplenomegaly, appearing in the first days of life, usually associated with fetal hydrops or fetal ascites. Early-infantile type is characterized by hepatosplenomegaly with neurological findings occurring between 2-24 months. Prolonged neonatal cholestatic icterus associated with progressive hepatosplenomegaly is the most common sign with spontaneous resolution of the icterus by 2-4 months of age. Main neurological symptoms are ataxia, vertical supranuclear gaze palsy (which is considered highly characteristic), dysarthria, dysphagia, dystonia, seizures, cataplexy and progressive dementia (8-10). As reported in the literature, our patient's findings (prolonged jaundice and hepatosplenomegaly which showed up in the second month of life) suggest that he had the early-infantile type of the disease. Neurological signs including psychomotor delay, focal seizures and mild spasticity appeared after 12 months. The mutation of NPC1 gene described in this study p.P733Sfs*10 (c.2196_2197insT) is a novel frameshift mutation leading to a stop codon (http://www.hgmd.org/). As the result is a truncated protein, this mutation most probably causes severe damage to its function. Wild Type protein has 1278 amino acids, while this truncated protein has just 743 amino acids. The disease has been reported with proteins with larger amino acid content. The disease management may include drugs and supportive therapy. Miglustat (ZavescaW, Actelion Pharmaceuticals Ltd, Switzerland), is a reversible inhibitor of glucosylceramide synthase, that has been shown to be effective in the treatment of progressive neurological manifestations in particularly among those with Late-infantile or Juvenile-onset disease, and has been used in that indication in Europe since 2010. It can be initiated as soon as neurological conditions occur. New therapies are in development based on experimental data on substrate reduction therapies (10). In our patient, no seizures were observed after the initiation of miglustat. Although he remained stable, sudden cardiac arrest occured at home. The exact cause of death could not be identified and an autopsy was recommended which the family refused. Laboratory data which were obtained a few days before his death, in an outpatient clinic showed elevated acute phase reactants, and normal blood gas and ammonia levels. Unfortunately, test to identify his metabolic state regarding isovaleric aciduria could not be performed.

When the clinical picture in a patient cannot be explained by the presence of one rare metabolic disease, it is essential to investigate further for the presence of other IEM's and more attention should be given in individuals with consanguineous parents. We also would like to emphasize the importance of NBS in the early detection of rare metabolic diseases and more care should be given for the extension of NBS programmes, especially in developing countries. A novel mutation for NPC has also been described.

\section{Ethics}

Informed Consent: Has been obtained.

Peer-review: Internally peer-reviewed.

\section{Authorship Contributions}

Surgical and Medical Practices: A.O., L.T., S.C., G.B., A.H., Concept: A.O., L.T., S.C., G.B., A.H., Design: A.O., L.T., S.C., G.B., A.H., Data Collection or Processing: A.O., L.T., S.C., G.B., A.H., Analysis or Interpretation: A.O., L.T., S.C., G.B., A.H., Literature Search: A.O., L.T., S.C., G.B., A.H., Writing: A.O., L.T., S.C., G.B., A.H.

Conflict of Interest: No conflict of interest was declared by the authors.

Financial Disclosure: The authors declared that this study received no financial support.

\section{References}

1. Bittles A. Consanguinity and its relevance to clinical genetics. Clin Genet 2001;60:89-98.

2. Applegarth DA, Toone JR, Lowry RB. Incidence of Inborn errors of metabolism in British Columbia. Pediatrics 2000;105:E10

3. Guy R, Forsyth JM, Cooper A, Morton RE. Co-existence of lysosomal storage diseases in a consanguineous family. Child Care Health Dev 2001;27:173-81.

4. Concolino D, Rapsomaniki M, Disabella E, et al. Co-existence of Phenylketonuria and Fabry disease on a 3 year-old boy: case report. BMC Pediatr 2010;10:32. Published online 2010.

5. Ensenauer R, Vockley J, Willard JM, et al. A common mutation is associated with a mild, potentially asymptomatic phenotype in patients with isovaleric acidemia diagnosed by newborn screening. Am J Hum Genet 2004;75:1136-42. Epub 2004 Oct 14

6. Mohsen AW, Anderson BD, Volchenboum SL, et al. Characterization of molecular defects in isovaleryl-CoA dehydrogenase in patients with isovaleric acidemia. Biochemistry 1998;37:10325-35.

7. Nasser I, Mohsen AW, Jelesarov I, et al. Thermal unfolding of medium chain acyl-CoA dehydrogenase and iso(3)valerylCoA dehydrogenase: study of the effect of genetic defects on enzyme stability. Biochim Biophys Acta 2004;1690:2232.

8. Vanier MT. Niemann-Pick disease type C. Orphanet J Rare Dis 2010;5:16.

9. Rodríguez-Pascau L, Toma C, Macías-Vidal J, et al. Characterisation of two deletions involving NPC1 and flanking genes in Niemann-Pick type C disease patients. Mol Genet Metab 2012;107:716-20.

10. Megías-Vericat JE, García-Robles A, Company-Albir MJ, et al. Early experience with compassionate use of 2 hydroxypropyl-beta-cyclodextrin for Niemann Picktype C disease: review of initial published cases. Neurol Sci 2017. 